\title{
MULTIFRAME SURE-LET DENOISING OF TIMELAPSE FLUORESCENCE MICROSCOPY IMAGES
}

\author{
Saskia Delpretti ${ }^{1}$, Florian Luisier ${ }^{1}$, Sathish Ramani ${ }^{1}$, Thierry Blu ${ }^{2}$ and Michael Unser ${ }^{1}$ \\ ${ }^{1}$ Biomedical Imaging Group, Ecole Polytechnique Fédérale de Lausanne, Switzerland \\ ${ }^{2}$ Department of Electronic Engineering, The Chinese University of Hong Kong, Hong Kong
}

\begin{abstract}
Due to the random nature of photon emission and the various internal noise sources of the detectors, real timelapse fluorescence microscopy images are usually modeled as the sum of a Poisson process plus some Gaussian white noise. In this paper, we propose an adaptation of our SURE-LET denoising strategy to take advantage of the potentially strong similarities between adjacent frames of the observed image sequence. To stabilize the noise variance, we first apply the generalized Anscombe transform using suitable parameters automatically estimated from the observed data. With the proposed algorithm, we show that, in a reasonable computation time, real fluorescence timelapse microscopy images can be denoised with higher quality than conventional algorithms.
\end{abstract}

Index Terms - Noise, Fluorescence, Wavelet, SURE

\section{INTRODUCTION}

Photon counting noise is the major source of noise in fluorescence imaging. Indeed, light emission, whether during illumination, observation or detection, is a random process where photons are emitted at random time intervals. This inherent statistical variation in the emission rate of photons is welldescribed by a Poisson process.

In addition to photon counting noise, signal-independent noise contributions also exist (e.g., electronic thermal noise, read-out noise, background noise,...). When all put together, these signal-independent noise contributions can be considered as normally distributed.

Denoising of such fluorescence images is currently an active area of research [1, 2]. In particular, wavelet-based [3] methods, especially in their redundant representations, have been proved to be very efficient for $2 \mathrm{D}$ image processing. However, redundant transformations become less attractive for data with larger size due to their high computational cost.

In this paper, our motivation is to come up with a both qualitatively and computationally efficient algorithm for de-

This work was supported by the Center for Biomedical Imaging (CIBM) of the Geneva - Lausanne Universities and the EPFL, the foundations Leenaards and Louis-Jeantet, as well as by the Swiss National Science Foundation under grant 200020-109415. noising timelapse (2D+time) fluorescence microscopy images. To achieve this goal, we introduce a time component in our recent 2D denoising strategy (orthonormal SURE-LET [4]), by taking into account the strong correlations between adjacent frames. This temporal sliding-window approach thus avoids the application of time-consuming 3D transformations by using neighboring frames as predictors of the current frame (see Fig. 1).

\subsection{Observation Model}

Similarly to [2], we consider that every observed pixels $y$ are i.i.d. random variables defined as:

$$
y=\alpha s+b
$$

where $s \sim \mathcal{P}(x)$ models the random nature of photon counting, $b \sim \mathcal{N}\left(\mu, \sigma^{2}\right)$ represents the signal-independent noise contribution and $\alpha>0$ symbolizes the overall gain of the photodetector.

Provided that the underlying intensities $x$ are sufficiently large (typically $x \geq 10$ ), the variance of the above Poisson-Gaussian mixture can be stabilized by the generalized Anscombe transform (GAT) [5]:

$$
\mathcal{A}(y)=\frac{2}{\alpha} \sqrt{\alpha y+\frac{3}{8} \alpha^{2}+\sigma^{2}-\alpha \mu}
$$

Then, the transformed pixels approximately obey $\mathcal{A}(y) \sim$ $\mathcal{N}(\mathcal{A}(\alpha x+\mu), 1)$, which makes the standard additive Gaussian white noise assumption relevant for denoising applications.

\subsection{Parameters estimation}

The knowledge of the parameters $\left(\alpha, \mu, \sigma^{2}\right)$ is essential for the application of the GAT (2). While $\mu$ and $\sigma^{2}$ may be measured by running separate calibration experiments [6], $\alpha$ may not be equivalent to the gain factor that is typically provided in microscopes and therefore must be estimated from the given data. For this, we devise a simple mechanism to estimate $\alpha$ and $\left(\sigma^{2}-\alpha \mu\right)$ based on the following identities:

$$
\begin{aligned}
& \mu_{y} \stackrel{\text { def }}{=} \mathscr{E}\{y\}=\alpha x+\mu \\
& \sigma_{y}^{2} \stackrel{\text { def }}{=} \operatorname{Var}\{y\}=\alpha^{2} x+\sigma^{2}
\end{aligned}
$$


This leads to the relationship:

$$
\sigma_{y}^{2}=\alpha \mu_{y}+\underbrace{\left(\sigma^{2}-\mu \alpha\right)}_{\beta} .
$$

To utilize (5), we first perform a segmentation of the noisy image stack into regions wherein the underlying noise-free signal is approximately constant. We then estimate $\mu_{y}$ and $\sigma_{y}^{2}$ by computing the sample-mean and sample-variance, respectively in each segment. Once that is done, we simply perform a robust linear regression on the set of points $\left(\mu_{y}, \sigma_{y}^{2}\right)$ : the slope yields an estimate of the gain $\alpha$ and the intercept at $\mu_{y}=0$ yields an estimate of $\beta$.

\section{MULTIFRAME SURE-LET}

The rationale behind our approach is that adjacent frames of fluorescence microscopy image sequences contain strong common information. In order to take into account these temporal correlations, we need to adapt our recent SURE-LET denoising algorithm [4].

\subsection{Principle}

In this section, $N$ denotes the number of pixels in an image, and $C$, the number of images in a sequence. We thus represent an image sequence $\mathrm{x}$ by a $C \times N$ matrix:

$$
\mathbf{x}=\left[\mathbf{x}_{1}, \mathbf{x}_{2}, \ldots, \mathbf{x}_{N}\right], \text { where } \mathbf{x}_{n}=\left[x_{n, 1}, x_{n, 2}, \ldots, x_{n, C}\right]^{\mathrm{T}}
$$

We consider that the distorting noise $\mathbf{b}$ is white, additive and independent from $\mathbf{x}$, and that it follows a multivariate Gaussian distribution whose $C \times C$ interframe covariance matrix is given by $\sigma^{2} \mathbf{I d}$. The observed noisy sequence is thus modeled as:

$$
\mathbf{y}=\mathbf{x}+\mathbf{b}
$$

We recall here the two key ingredients of the SURE-LET approach devised in [4] and show their adaptation to multiframe denoising:

1. Stein's unbiased risk estimate (SURE) [7]: in denoising, the most popular measure of quality is the meansquared error (MSE) between the original signal $\mathbf{x}$ and the denoised one $\hat{\mathbf{x}}$. In real applications, we can obviously not compute this actual MSE since it involves the unknown noise-free data. We propose instead to minimize an unbiased estimate of the MSE, known as SURE. For multiframe processing $\hat{\mathbf{x}}_{n}=\boldsymbol{\theta}\left(\mathbf{y}_{n}, \mathbf{p}_{n}\right)$ involving the noisy data $\mathbf{y}_{n}$ and any other information $\mathbf{p}_{n}$ statistically independent of $\mathbf{y}_{n}$, SURE becomes:

$$
\begin{aligned}
\epsilon= & \frac{1}{C N} \sum_{n=1}^{N}\left\|\boldsymbol{\theta}\left(\mathbf{y}_{n}, \mathbf{p}_{n}\right)-\mathbf{y}_{n}\right\|^{2}+ \\
& \frac{2 \sigma^{2}}{N} \sum_{n=1}^{N} \operatorname{Tr}\left\{\nabla_{\mathbf{y}_{n}} \boldsymbol{\theta}\left(\mathbf{y}_{n}, \mathbf{p}_{n}\right)\right\}-\sigma^{2}
\end{aligned}
$$

2. A linear expansion of thresholds (LET): to take full advantage of the quadratic form of $\epsilon$ w.r.t. $\boldsymbol{\theta}$, we use a thresholding function that is a linear combination of elementary non-linear denoising functions $\boldsymbol{\theta}_{k}$, with unknown $C \times C$ weights $\mathbf{a}_{k}$ :

$$
\boldsymbol{\theta}\left(\mathbf{y}_{n}, \mathbf{p}_{n}\right)=\sum_{k=1}^{K} \mathbf{a}_{k}^{T} \boldsymbol{\theta}_{k}\left(\mathbf{y}_{n}, \mathbf{p}_{n}\right)
$$

With the above thresholding function, the search for the parameters $\mathbf{a}_{k}$ that minimize the SURE derived in (7) simply amounts to solving a linear system of equations.

Note that these theoretical adaptations $(7,8)$ are similar to those encountered in [8] for multichannel denoising, with the noteworthy difference that, for multiframe denoising, $C$ (odd) adjacent frames are considered to denoise the central $c=(C+1) / 2$ frame, as illustrated in Fig. 1. The parameters of (8) have therefore to be optimized for this central frame only.

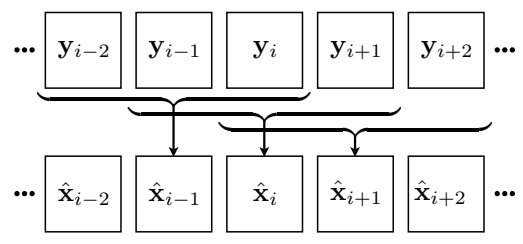

Fig. 1. Use of adjacent frames for multiframe denoising.

\subsection{The Algorithm}

The GAT (2) is first applied to the raw image sequence. Then, for each frame, a 2D orthonormal wavelet transform (OWT) [3] is performed and the stabilized noise standard deviation $\sigma_{\text {stab }}$ is estimated in the highest frequency subband using the robust MAD estimator [9].

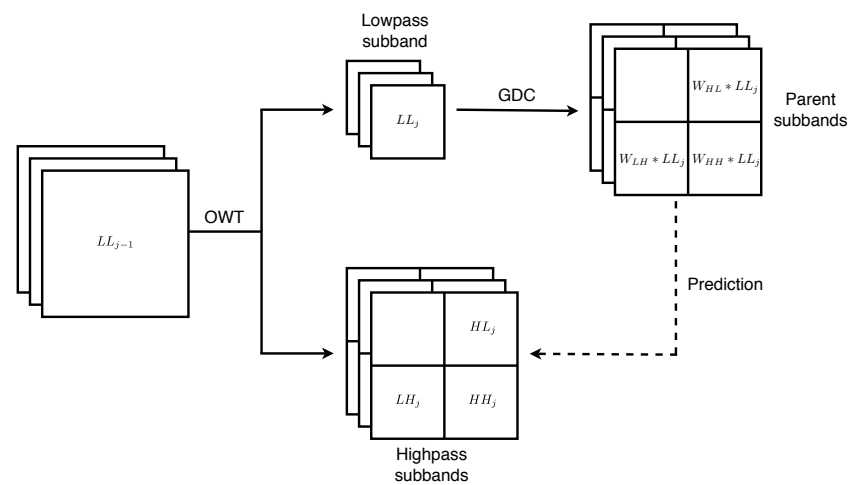

Fig. 2. Multiframe illustration of the group delay compensation (GDC). The GDC is obtained by appropriately filtering the same scale lowpass subband.

Thanks to the orthogonality of the wavelet transform, the noise model (6) is still valid inside every wavelet subbands, 
and their respective noise components are statistically independent. Therefore, we propose to build $\mathbf{p}_{n}$ out of the group delay compensated [4] lowpass subband at the same scale (see Fig. 2).

These interscale predictors $\mathbf{p}_{n}$ are then used as discriminators between zones of high magnitude and low magnitude (i.e. mainly due to noise) wavelet coefficients, to give the following interscale multiframe thresholding:

$$
\begin{aligned}
\boldsymbol{\theta}\left(\mathbf{y}_{n}, \mathbf{p}_{n}\right)= & \underbrace{\gamma\left(\mathbf{p}_{n}^{\mathrm{T}} \mathbf{R}^{-1} \mathbf{p}_{n}\right) \gamma\left(\mathbf{y}_{n}^{\mathrm{T}} \mathbf{R}^{-1} \mathbf{y}_{n}\right)}_{\text {small parents and small coefficients }} \mathbf{a}_{1}^{\mathrm{T}} \mathbf{y}_{n}+ \\
& \underbrace{\bar{\gamma}\left(\mathbf{p}_{n}^{\mathrm{T}} \mathbf{R}^{-1} \mathbf{p}_{n}\right) \gamma\left(\mathbf{y}_{n}^{\mathrm{T}} \mathbf{R}^{-1} \mathbf{y}_{n}\right)}_{\text {large parents and small coefficients }} \mathbf{a}_{2}^{\mathrm{T}} \mathbf{y}_{n}+ \\
& \underbrace{\gamma\left(\mathbf{p}_{n}^{\mathrm{T}} \mathbf{R}^{-1} \mathbf{p}_{n}\right) \bar{\gamma}\left(\mathbf{y}_{n}^{\mathrm{T}} \mathbf{R}^{-1} \mathbf{y}_{n}\right)}_{\text {small parents and large coefficients }} \mathbf{a}_{3}^{\mathrm{T}} \mathbf{y}_{n}+ \\
& \underbrace{\bar{\gamma}\left(\mathbf{p}_{n}^{\mathrm{T}} \mathbf{R}^{-1} \mathbf{p}_{n}\right) \bar{\gamma}\left(\mathbf{y}_{n}^{\mathrm{T}} \mathbf{R}^{-1} \mathbf{y}_{n}\right)}_{\text {large parents and large coefficients }} \mathbf{a}_{4}^{\mathrm{T}} \mathbf{y}_{n}
\end{aligned}
$$

where $\left\{\begin{array}{l}\gamma(x)=\exp \left(-\frac{|x|}{12 C}\right) \\ \bar{\gamma}(x)=1-\gamma(x)\end{array}\right.$ are the discriminative functions.

The denoised sequence is finally obtained by performing the inverse wavelet transform on the processed coefficients and the inverse GAT on the reconstructed images.

\section{EXPERIMENTS}

\subsection{Validation on simulated data}

We applied the proposed algorithm on a simulated sequence of size $496 \times 400 \times 10$ with a mean intensity of 16.14 . We then degraded this sequence with a signal-dependent Poisson noise with gain $\alpha=1$ and an additive zero-mean Gaussian white noise of variance $\sigma^{2}=25$, which gave an input signalto-noise ratio (SNR) of $9.25 \mathrm{~dB}$ (see Fig. 3). By applying the procedure described in Section 1.2, we got the estimated noise parameters: $\alpha=0.98$ and $\beta=24.9$. After the GAT, the estimated noise standard deviations $\sigma_{\text {stab }}$ lied in the range of $[1.00,1.02]$ for the whole sequence. Using these estimated parameters, we got an output SNR of $25.78 \mathrm{~dB}$ in $15 \mathrm{~s}$ using $C=3$ adjacent frames. To compare with, we obtained a SNR of $24.40 \mathrm{~dB}$ in $5 \mathrm{~s}$ with the monoframe $(C=1)$ restriction of our algorithm and a SNR of $22.57 \mathrm{~dB}$ in $2 \mathrm{~s}$ when applying the SUREshrink [9] independently inside each frame.

\subsection{Results on real data}

We show the result of our SURE-LET algorithm applied on a real timelapse fluorescence microscopy image sequence ${ }^{1}$ of C. Elegans embryos ${ }^{2}$. The embryos were imaged with an Olympus IX 81 motorized inverted microscope equipped with

\footnotetext{
${ }^{1}$ Acquired with the invaluable help of the BIOP team at EPFL.

${ }^{2}$ Kindly provided by Prof. Pierre Gönczy, UPGON, ISREC, EPFL.
}
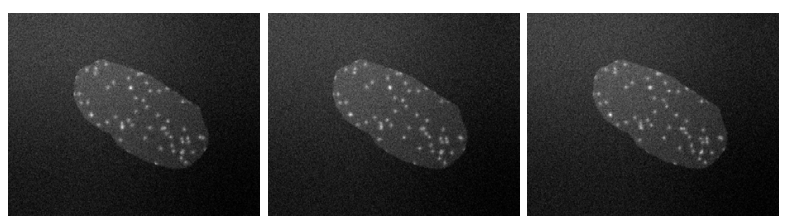

Fig. 3. 3 adjacent frames of the simulated noisy sequence.

a spinning disk Perkin Elmer Ultra View ERS. 1000 frames were imaged at $2 \mathrm{~Hz}$ for a total duration of $8 \mathrm{~min} 20 \mathrm{sec}$. Frame number 40 is displayed in Fig. 4(A).

In this special worm line, a GFP encoding cassette was inserted under the promoter of tubulin, a major cell cytoskeleton component active during cellular division. The green fluorophore thus tags the tubulin molecules in the embryos. More precisely, we see two C. Elegans embryos at different stages of early development. The lower embryo has just gone through its very first division, whereas the upper embryo has already gone through multiple divisions.

We obtained the following noise parameters: $\alpha=8.72$ and $\beta=-3770.17$. Using these values for the GAT gave a stabilized noise standard deviation $\sigma_{\text {stab }}$ in the range of $[0.98,1.02]$, which confirmed the considered observation model (1). We considered $C=5$ adjacent frames and four iterations of an OWT using Daubechies' symlets filters with eight vanishing moments [3].

As can be seen in Fig. 4, the denoising quality of the proposed algorithm (D) is much higher than standard procedures such as the median filtering (B) or the wavelet-based SUREshrink (C). Many relevant details are extracted from the noisy image (A). Polar bodies that were ejected during meiosis get clearly visible as two different entities in the lower embryo, whereas as a single entity with the conventional algorithms. The linear shape of microtubules appears more clearly, in a star-like disposal from the centrosome to the periphery in the right cell and globally dispersed in the left cell of the lower embryo, enabling both cells to be visually easily separable from one another. In the upper embryo, the various cells can now be distinguished from one another, with internal filamentous structure appearing in each cell. Finally, the egg shell that is surrounding both embryos gets clearly visible with the proposed algorithm, whereas hardly distinguishable with conventional algorithms.

\section{CONCLUSION}

Thanks to an efficient integration of adjacent frames dependencies in our SURE-LET denoising strategy, we show that, in a reasonable computation time, real timelapse fluorescence microscopy images can be denoised with higher quality than conventional algorithms. 
(A)

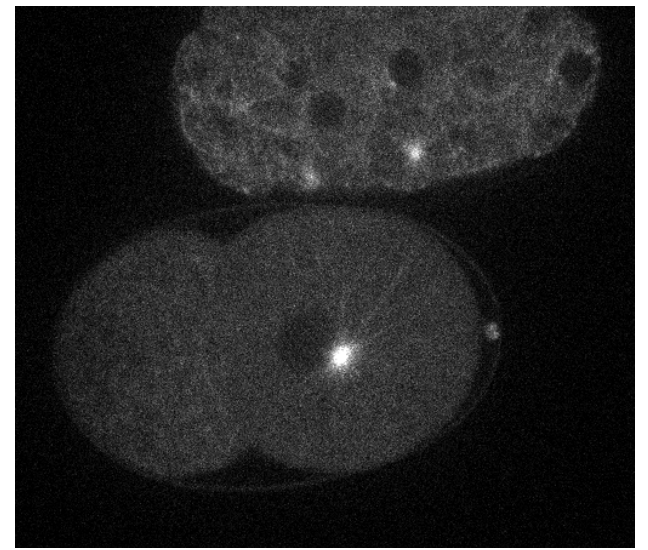

(C)

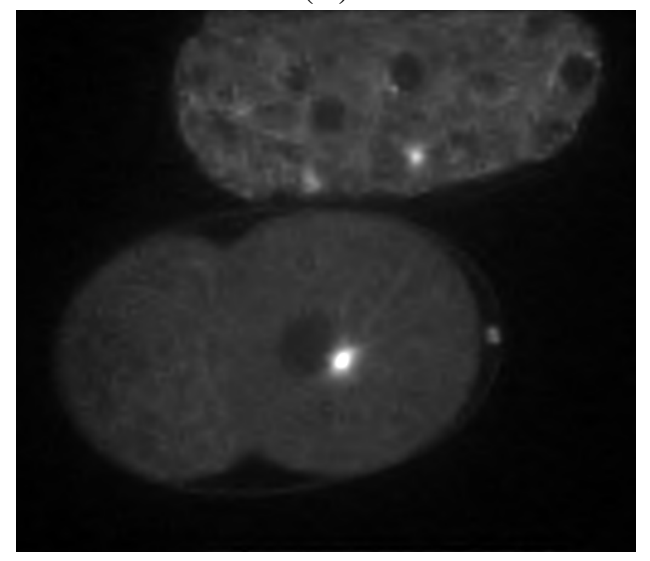

(B)

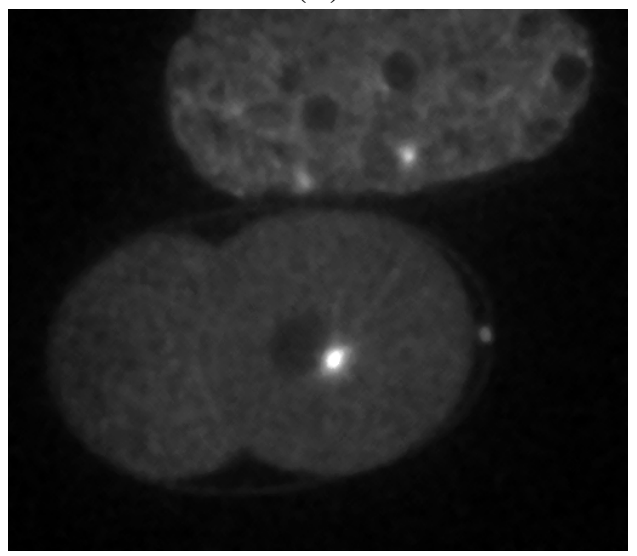

(D)

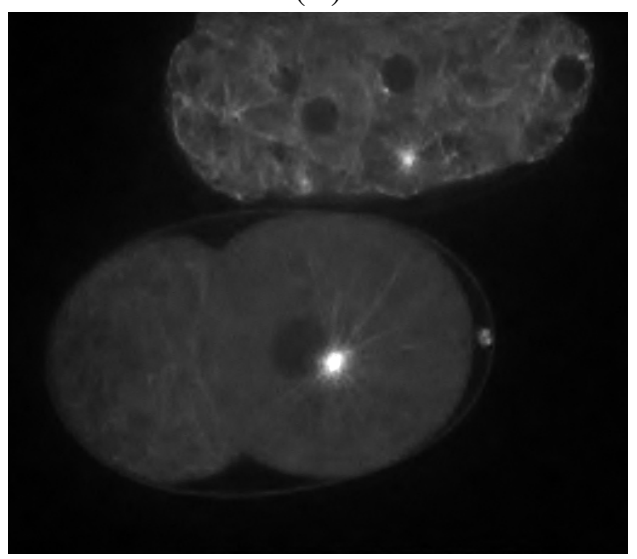

Fig. 4. One particular frame (at $t=20$ s) of the fluorescence sequence. (A) Raw data. (B) Median filter. (C) SUREshrink. (D) The proposed algorithm.

\section{REFERENCES}

[1] J. Boulanger, C. Kervrann, and P. Bouthemy, “An Adaptive Statistical Method for Denoising 4D Fluorescence Image Sequences with Preservation of Spatio-temporal Discontinuities," IEEE International Conference on Image Processing, vol. 2, pp. 145-148, 2005.

[2] B. Zhang, M. J. Fadili, J.-L. Starck, and J.-C. OlivoMarin, "Multiscale Variance-stabilizing Transform for Mixed-Poisson-Gaussian Processes and its Applications in Bioimaging," IEEE International Conference on Image Processing, vol. 6, pp. 233-236, 2007.

[3] I. Daubechies, "Ten Lectures on Wavelets," CBMS-NSF Regional Conference series in Applied Mathematics, vol. 61 of Proc., March 1992.

[4] F. Luisier, T. Blu, and M. Unser, "A New SURE Approach to Image Denoising: Interscale Orthonormal Wavelet Thresholding," IEEE Transactions on Image Processing, vol. 16, no. 3, pp. 593-606, March 2007.
[5] F. Murtagh, J.-L. Starck, and A. Bijaoui, "Image Restoration with Noise Suppression using a Multiresolution support," Astron. Astrophys. Suppl. Ser., vol. 112, pp. 179189, 1995.

[6] D. L. Snyder and A. M. Hammoud, "Image Recovery from Data Acquired with a Charge-Coupled-Device Camera," Journal of Optical Society of America A, vol. 10, no. 5, pp. 1014-1024, 1993.

[7] C. Stein, "Estimation of the Mean of a Multivariate Normal Distribution," The Annals of Statistics, vol. 9, pp. 1135-1151, 1981.

[8] F. Luisier and T. Blu, "SURE-LET Multichannel Image Denoising: Interscale Orthonormal Wavelet Thresholding," To appear in IEEE Transactions on Image Processing.

[9] D. L. Donoho and I. M. Johnstone, "Adapting to Unknown Smoothness via Wavelet Shrinkage," Journal of the American Statistical Association, vol. 90, no. 432, pp. 1200-1224, December 1995. 